\title{
MICOTA AMBIENTAL EN LA UNIDAD DE TERAPIA INTENSIVA DE UN HOSPITAL PEDIATRICO
}

\author{
(Ambiental mycota at an intensive care unit of a pediatric hospital)
}

\author{
Mariana Fernández, Magdalena Mangiaterra, Gustavo Giusiano \\ Departamento Micología. Instituto de Medicina Regional. \\ Universidad Nacional del Nordeste. Av. Las Heras 727, 3500 Resistencia, Argentina. \\ E-mail: gustavogiusiano@yahoo.com.ar
}

Palabras clave: Hongos ambientes internos, unidades de terapia intensiva.

Key words: Indoor airborne fungi, intensive care unit.

\section{RESUMEN}

En las últimas décadas se ha demostrado claramente el rol del aire en la transmisión de microorganismos oportunistas en ambientes endógenos, especialmente en centros hospitalarios, personal dependiente $y$ en pacientes susceptibles hospitalizados. El presente trabajo tiene como finalidad detectar la cantidad y diversidad de la micobiota anemófila y de superficies presente en la Unidad de Terapia Intensiva (UTI) del Hospital Pediátrico "Juan Pablo II» de la ciudad de Corrientes (Argentina), mediante un Colector SAS súper 100 y por el método del hisopo, en 2 períodos estacionales (otoño y primavera), cada 15 días, a mediodía y después de la limpieza habitual de la unidad. El número de UFC hallado, excedió considerablemente los parámetros establecidos y los géneros de hongos filamentosos más frecuentes en el aire y en las superficies fueron los integrantes de los géneros: Cladosporium, Penicillium y Aspergillus, seguidos por Acremonium, Fusarium, Curvularia y Chrysonilia. Algunos de sus integrantes son considerados como patógenos oportunistas y productores de toxinas como: Aspergillus fumigatus, A.flavus, A.niger, Acremonium strictum complex, Fusarium oxysporum, Trichoderma harzianum y Stachybotrys atra, (este último se presentó una sola vez). La presencia de estos taxa se considera inaceptable en ambientes cerrados.

Debido a la información obtenida en el presente trabajo se intensificaron las medidas para disminuir la carga fúngica ambiental de esta zona hospitalaria donde están bajo tratamiento intensivo pacientes en alto riesgo.

Recibido el 24 de Noviembre 2009

Aceptado el 28 de Diciembre 2009

\section{ABSTRACT}

Lately decades have clearly evidenced the role that air plays in the transmission of opportunistic microorganisms under endogenous environments, mainly in hospitals, health centers, working personnel as well as in liable patients that are hospitalized. The present paper has been designed to detect the amount and diversity of anemophyle and surface mycota occurring at the Intensive Care Unit (UTI) in the Hospital Pediátrico "Juan Pablo II» of the city of Corrientes (Argentina) by means of a super 100 SAS Colector and using the sprinkling water method in 2 seasonal periods (fall and spring), every 15 day, at midday and after the regular cleaning process of the unit. The number of UFC found exceeded greatly the established parameters whilst filamentous fungi occurring most frequently in the air and surfaces were those belonging to the genera: Cladosporium, Penicillium and Aspergillus, followed by Acremonium, Fusarium, Curvularia and Chrysonilia. Some of their members are considered opportunistic pathogenus and toxin producers such as Aspergillus fumigatus, A.flavus, A.niger, Acremonium strictum complex, Fusarium oxysporum, Trichoderma harzianum and Stachybotrys atra, (the latter appeared only once). The presence of these taxa is found to be unacceptable indoors.

Considering the information described in this paper, control measures were intensified to reduce environmental fungal load in this hospital area where patients under high risk are treated.

\section{INTRODUCCION}

Cuando un paciente ingresa a una unidad de terapia intensiva (U T I), sus familiares confían en que, por lo 
menos, estará resguardado de los microorganismos oportunistas que coexisten en el ambiente. Sin embargo, las estadísticas demuestran que el número de muertes derivadas de infecciones por hongos contraídas en las salas de cuidados especiales alcanza hoy cifras no despreciables (1).

La causa de esto es que el aire que respiramos no es una simple mezcla de nitrógeno, oxígeno y dióxido de carbono con vapor de agua. En suspensión se encuentran diversas partículas inertes de polvo, humos, otras impurezas y una rica biota constituida por ácaros, polen, propágulos fúngicos y bacterias. Los bioaerosoles son formados por partículas de origen biológico o con actividad biológica que pueden afectar a los seres vivos mediante procesos infecciosos, alérgicos, irritantes y tóxicos. Las infecciones pueden ser causadas por: virus, bacterias y hongos; las alergias y las irritaciones por: polen, hongos y sus propágulos de dispersión y, finalmente, las intoxicaciones por toxinas bacterianas y micotoxinas $(1,2,3,4,5,6)$.

En la práctica médica tienen cada vez más importancia las enfermedades causadas por hongos oportunistas adquiridas en ambientes hospitalarios. Entre los agentes más destacados se encuentran los integrantes de los géneros, Aspergillus, Candida, Penicillium, Fusarium, Cryptococcus, Alternaria, Cladosporium, Acremonium, Stachybotrys, Curvularia, Phoma, entre otros. La mayor gravedad se presenta en los lugares de alto riesgo, como guarderías, residencias de ancianos, quirófanos y unidades de terapia intensiva (5).

En los ambientes internos la proliferación de los contaminantes biológicos (hongos sobretodo) se presenta en zonas húmedas, es decir, humedad de las paredes, agua estancada procedente de aparatos de refrigeración, humidificadores, floreros, filtros de aire, alfombras, cortinas de tela, macetas con plantas o pinturas porosas. Por lo tanto, la vigilancia epidemiológica en los hospitales es necesaria para evitar enfermedades producidas por estos organismos, tales como sinusitis fúngica, colonización de heridas o infecciones invasoras que se caracterizan por tener alta morbimortalidad $(6,7,8)$. Para ello, es imprescindible conocer la calidad del aire confinado, realizando un recuento selectivo de levaduras y de mohos, como el más elemental mecanismo de prevención de enfermedades ( 5 , $9,10)$.

Los hongos, por su ubicuidad y particular biología, influyen directa e indirectamente en la economía. Las técnicas para el estudio de la biota fúngica aérea han sido desarrolladas para conocer la dispersión de los hongos que son patógenos u oportunistas para el ser humano y los que lo son para las plantas $(11,12)$.

En todos los ambientes considerados de riesgo, el control microbiológico del aire debe hacerse por un método activo o volumétrico y por un método pasivo que puede ser la sedimentación o el hisopado. El muestreo activo constituye un instrumento útil para recoger información sobre el número de microorganismos suspendidos en el aire y el pasivo para valorar la cantidad de microorganismos que se pueden depositar en una herida o sobre el instrumental médico-quirúrgico $(9,13)$.

El objetivo del presente trabajo fue conocer la carga y la diversidad de la biota fúngica ambiental de la UTI de un hospital pediátrico.

\section{MATERIALES Y METODOS}

Las muestras se tomaron en la UTI del Hospital Pediátrico «Juan Pablo II» de la ciudad de Corrientes, Argentina. La sala se encuentra en el primer piso, mide 13 x $20 \mathrm{~m}$, con el eje mayor orientado en sentido norte-sur. Sobre las paredes del Este y el Oeste hay ventanas con vidrios fijos. En el centro de la sala está el sector de enfermería, limitado por una pared de 1,20 m de alto. En la sala hay cinco piletas de lavado, tres sobre la pared Este, una sobre la pared Oeste y otra dentro del sector de enfermería.

La recolección de los propágulos fúngicos se realizó cada 15 días, durante la primavera y el otoño, con el colector de aire Surface Air Sampler, P.B.I. Intenational super 100 (SAS).

En todas las muestras se siguió un diseño diagonal (13), tomando 5 puntos, 4 en los extremos y uno en el sector enfermería. Con el fin de comparar, tanto la carga como la diversidad fúngica, se muestreó el ambiente inmediato a la puerta de acceso, que se consideró como blanco (Bl) (Figura 1). Las muestras se tomaron al mediodía, después de la limpieza habitual de la sala. El SAS se colocó a 1,50 metros del suelo y el flujo de aire fue de 200 litros/ min. Como medio de impacto se utilizó agar papa dextrosa con cloranfenicol 250mg/L (PDA/ATB), en placas de Petri de $9 \mathrm{~mm}$.

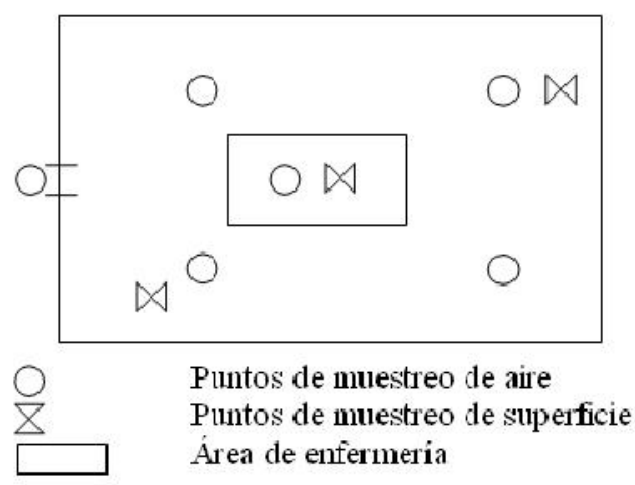

Figura 1. Plano de la UTI pediátrica y zonas de muestreos 
Las muestras de superficie (Sup) se tomaron de los mesones del sector enfermería y de los aparatos de control de 2 camas (Figura 1) por el método del hisopo (13). En ese momento se sembró por estría en PDA/ATB, contenido en placas de Petri de $9 \mathrm{~mm}$ (1).

La toma de muestra se realizó simultáneamente por los dos métodos y las placas obtenidas de ambos tipos de muestreo se incubaron a $28^{\circ} \mathrm{C}$ durante $72 \mathrm{~h}$; al cabo de la cuales se realizó el recuento de las unidades formadoras de colonia por placa (UFC/placa).

Para determinar las unidades formadoras de colonias por metro cúbico (UFC/m3), conociendo el número de colonias por placa ( $\mathrm{NC}$ ), el flujo de aire y el tiempo de muestreo que se ha usado (NU), se aplica la siguiente fórmula (12):

$$
\mathrm{N}^{\mathrm{o}} \mathrm{UFC} / \mathrm{m}^{3}=\frac{\mathrm{NC} \times 1000}{\mathrm{NU}}
$$

Cada taxa se contabilizó una sola vez en cada muestra, no importando si se repetía en la misma placa o aparecía en las otras.

Las cepas de mohos se identificaron por su macro y micromorfología. En los casos en que fue necesario se repicó en medios de cultivo especiales y se realizaron microcultivos para llegar a la tipificación definitiva. Las colonias que no desarrollaron órganos de fructificación en los subcultivos, tras 20 días de incubación a $28^{\circ} \mathrm{C}$, fueron agrupadas como micelio sin fructificación.

Para la clasificación taxonómica de los hongos se utilizaron las claves taxonómicas de: Nelson et al. (1983); Gams (1971); Onions et al. (1981); Von-Arx (1981); de Hoog et al. (2000); Samson et al. (2000); Rifai (1969); Bissett (1991); Piontelli \& Toro (1994); Sivanesan (1987); Pitt (1986); Ellis \& Ellis (1987); Klich \& Pitt (1988); Carmichael et al. (1980) $(14,15,16,17,18,19,20,21,22,23,24,25,26,27)$.

La correlación estadística de variables discretas se estudió mediante el test de Student y la técnica de Chi cuadrado, considerándose un nivel de significancia de $\mathrm{p}$ $<0,05$. Se trabajó con el programa Epi info versión 6. Para el estudio de diversidad se aplicó el Índice de ShannonWiener.

\section{RESULTADOS}

Se tomaron 6 muestras en otoño y 6 en primavera, sin embargo, el quinto muestreo de otoño fue posterior a la fumigación de la sala, por lo tanto crecieron pocas colonias y en el tercer muestreo de primavera, la presencia de Chrysonilia sitophila (similis) en todas las placas, impidió el recuento e identificación de otros hongos, pues las placas fueron eliminadas para evitar la contaminación del laboratorio y otros ambientes.

El recuento promedio del total de colonias en UTI en otoño fue de $557 \mathrm{UFC} / \mathrm{m}^{3}$ y en primavera de $336 \mathrm{UFC} /$ $\mathrm{m}^{3}$. No se observaron diferencia significativa entre estos recuentos. En el Bl (puerta de acceso a la UTI) el recuento promedio en otoño fue de $568 \mathrm{UFC} / \mathrm{m}^{3}$ y en primavera de $420 \mathrm{UFC} / \mathrm{m}^{3}$. No se observaron diferencias significativas entre estos recuentos en ambas estaciones

En las superficies, el recuento promedio de UFC/placa fue de 30,8 UFC/placa en otoño y de $47 \mathrm{UFC/}$ placa en primavera. No se observaron diferencias significativas entre ellos.

Considerando aire y superficies se aislaron 324 colonias de hongos de las que se distinguieron 47 levaduras, 11 micelios dematiáceos sin fructificación, 17 micelios hialinos sin fructificación, 7 formas picnidiales y se identificaron 19 géneros y 52 especies (Tabla 1).

La zona blanco $(\mathrm{Bl})$ presentó el menor número de aislados en ambas estaciones, el género Penicillium se vió representado escasamente y la especie P.citrinum destacó solo en otoño, junto a 4 especie de Aspergillus (entre ellos A.fumigatus). Se destacan por su frecuencia en ambos períodos, C.cladosporioides complex, Alternaria alternata, Curvularia lunata, y Chr. Sitopila (similis) (Tabla 1).

Los géneros más frecuentes en número de especie en ambas estaciones y zonas de muestreo fueron Penicillium y Aspergillus, sin embargo, Aspergillus presentó mayor frecuencia de presencia (21,32\%) que Penicillim, $(17,49 \%)$, seguido por el conjunto de levaduras blancas, rosadas y negras $(15,22 \%)$ y Cladosporium cladosporioides complex $(9,72 \%)$ Estos 4 grupos de taxa representaron el $63,75 \%$ de todos los aislamientos (Tabla 1 ).

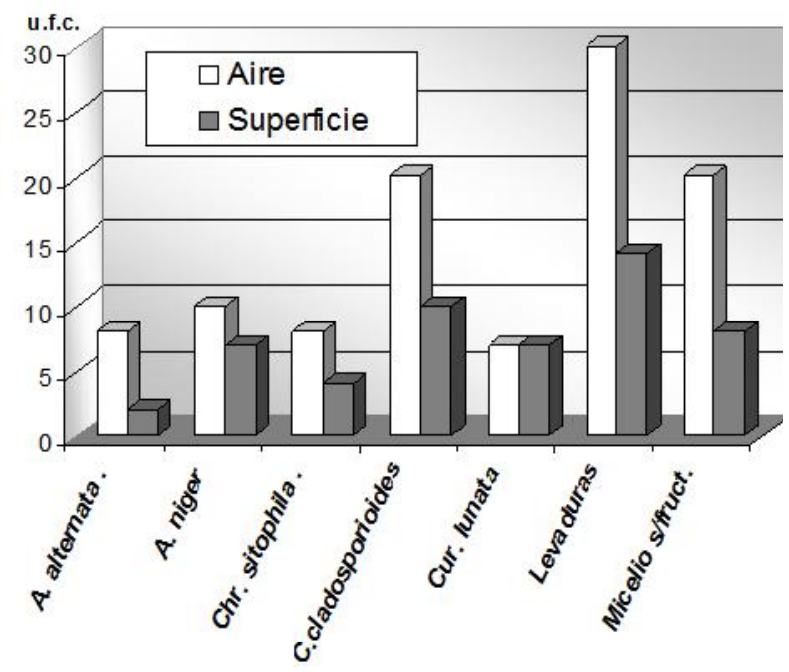

Figura 2. Especies más aisladas y constantes en todos los lugares de muestreo y estaciones 
Tabla 1. Freeueneia de especies aisladas en el aire y superficies, discriminadas por estación.

\begin{tabular}{|c|c|c|c|c|c|c|}
\hline \multirow[t]{3}{*}{ Taxa y categorias } & \multicolumn{6}{|c|}{ I nidad de Terapia Intensiva } \\
\hline & \multicolumn{3}{|c|}{ Otoño } & \multicolumn{3}{|c|}{ Primavera } \\
\hline & Blanco & $1 \mathrm{TI}$ & Superficie & Blanco & ITI & Superficie \\
\hline Acremonium kiliense Girtity & & 2 & & 2 & & 1 \\
\hline Acremoniam strictum complex W. Gams & 1 & 4 & 3 & & & \\
\hline Acremonuim spp. & & 1 & 1 & & & \\
\hline Alternaria alternata complex Keissler & 3 & 3 & & 1 & 1 & 2 \\
\hline Alternaria temuissima Wiltsh & & & 1 & & & \\
\hline Aspergillus flavers Linh. & 1 & 2 & & 2 & & \\
\hline Aspergillus fumiganus complex If. & 1 & 3 & & 1 & & \\
\hline Aspergillus nidulans (Eidam) Winter & & & 1 & & & \\
\hline Aspergillus niger van Tieghem & 2 & 4 & 2 & 1 & 3 & 5 \\
\hline Aspergillus ochracens Wilhem & & 2 & & & 2 & \\
\hline Aspergillus parasiticus Spcare & & & 1 & & & \\
\hline Aspergillus spe. & & 2 & & & 1 & 1 \\
\hline Aspergillus svdowii (Bain. \& Sart) Thome Church & & 4 & 2 & & 5 & 2 \\
\hline Aspergillus terreus Thom & & 2 & & & 2 & \\
\hline Aspergillus ustus (Bain.) Thom\& Church & & & & 2 & 3 & 1 \\
\hline Aspergillus versicolor (Vuill.) Tirahoschi & 1 & 2 & . & & & \\
\hline Aspergillus unguis (Emile-Weil \& Gaudin) Dodge & & & & & 2 & \\
\hline Botryotrichum piluliferum Sace. \& March & & 1 & & & & \\
\hline Chrysonilia sitophila (similis) (Mont.) v. Arx & 2 & 4 & 3 & 1 & 1 & 1 \\
\hline Cladosporium cladosporioüles (Fres.) Vries & 5 & 5 & 5 & 5 & 5 & 5 \\
\hline Curvularia Iunata (Wakker) Boedijn & 2 & 4 & 4 & 1 & & 3 \\
\hline Epiccocum purpurascens Ehrenb Fx Schlecht & & 1 & & & & \\
\hline Fusarisum chtamydosporum Wollenw \& Reinking & & 2 & & & & \\
\hline Fusarium oxysporum Schlocht. & 2 & 3 & 1 & & & \\
\hline Fusarium semitectum Berh.\&Rav. & 1 & & & & 1 & \\
\hline Fusarium solani (Mart.) Sacc. & 1 & & & i & & 2 \\
\hline Fusarium subglutinans (Wollenw \&Reink.) Nelson & & & & 1 & & \\
\hline Gilmaniella humicola Barron & & 1 & 1 & & & \\
\hline Levadura amarilla & & 1 & & & & \\
\hline 1.evadaras blancas & 2 & 6 & 6 & 4 & 3 & 5 \\
\hline Levaduras negras & & 1 & & & 2 & 1 \\
\hline Levaduras rojas & 1 & 6 & 2 & & 5 & 2 \\
\hline Micelio demateaceo s/ fructificación & $i$ & 4 & 1 & 1 & 2 & 2 \\
\hline Micelio hialino s/ fructificación & 4 & 4 & 2 & 2 & 2 & 3 \\
\hline Mucor racemosus lins. & & & 1 & & & 1 \\
\hline Paecilontyces marquandii (Massee) Ilughes & & & & & 1 & \\
\hline Penicillium brevicompacfum Diereks & & & & & 2 & 1 \\
\hline Penicillium canescens Sopp & & 1 & & & & \\
\hline Penicillium chermesimum Biourge & & 1 & & 1 & & 1 \\
\hline Penicillium chrvsogenum Thom & & 1 & 1 & & 1 & \\
\hline Penicillium citrinum Thom & 4 & 4 & ' & & 2 & \\
\hline Penicillium corylophilum Dierchx & & 1 & & & & \\
\hline Penicillium fellutanum Biourge & & 1 & 1 & & & \\
\hline Pentcillium glabrum (Welmer) Westling & & 1 & & 1 & 3 & 1 \\
\hline Penicilfium implicatum Biourge & & & & & 1 & \\
\hline
\end{tabular}


(Continuación Tabla 1.)

\begin{tabular}{|c|c|c|c|c|c|c|}
\hline Penicillium oxalicum Currie\&Thom & & 1 & 1 & & 1 & 1 \\
\hline Penicillium pinophilum Hedgcock & & 2 & & & 1 & \\
\hline Penicillium simplicissimum (Oudem)Thom & & & & & 1 & \\
\hline Penicillium spinulosum Thom & & 2 & 2 & & 1 & \\
\hline Penicillium $\mathrm{spp}$ & & 1 & & 1 & 1 & 1 \\
\hline Penicilliun variabile Sopp & & 2 & 1 & & & 1 \\
\hline Pithomyces chartarum (Berk.\&Curt) MB.Ellis & & & & & & 1 \\
\hline Pienidios & & & 2 & 1 & 1 & \\
\hline Rhizopus oryzae Went\& Prinsen Geerlings & & & 1 & & & \\
\hline Rhizopus stolonifer (Ehrenb.Fr.)Vuill. & & & & & 1 & 1 \\
\hline Scytalidium hyalinum Campbell\&Mulder & & 1 & & & & \\
\hline Stachybotrys atra (Ehrenb. ExLink) Hughes & & & 1 & & & \\
\hline Trichoderma sec. longibrachiatum Rifai & & & & & 2 & \\
\hline Trichoderma harzianum Rifai & 1 & 1 & 2 & 2 & 2 & \\
\hline Trichoderma koningii Oudem. & & & 1 & & 1 & \\
\hline Trichoderma spp. & & 1 & 1 & & & \\
\hline Trichothecium roseum Link & & & & & & 1 \\
\hline Total sector & 35 & 96 & 52 & 30 & 64 & 47 \\
\hline Total estación & \multicolumn{3}{|c|}{183} & \multicolumn{3}{|c|}{141} \\
\hline Total Unidad & \multicolumn{6}{|c|}{324} \\
\hline
\end{tabular}

Las especies más aisladas y constantes en todos los lugares de muestreo y ambas estaciones, fueron: Cladosporium cladosporioides complex (30 aislamientos), junto a Aspergillus niger (17), Chrysonilia sitophila (similis) (12), levaduras blancas (26) y los micelios hialinos y dematiáceos sin fructificar (28). Todos ellos corresponden a más de un tercio $(34,87 \%)$ del total de aislamientos (Figura 2, Tabla 1).

Las especies que aparecieron en UTI exclusivamente en primavera fueron 11, donde se destaca: Aspergillus ustus, A. unguis y Trichoderma secc. Longibrachiatum, los restantes tuvieron escasa representatividad mientras en otoño fueron 19: siendo Acremonium strictum complex, Fusarium oxysporum y $\boldsymbol{A}$. versicolor las más frecuentes (Tabla 1).

Las especies que aparecieron en las superficies exclusivamente en primavera fueron 13 , siendo las de mayor frecuencia: Alternaria alternata complex y $\boldsymbol{F}$. solani complex, mientras en otoño fueron 17, con la mayor frecuencia de: $\boldsymbol{A}$. strictum complex, P. spinulosum y Trichoderma harzianum complex (Tabla 1).

La diversidad general según el índice de ShannonWiener fue de 2,8. El mismo índice mostró que la diversidad entre UTI y Bl es notoria. En UTI fue de 2,0 y en el Bl de 1,2. La diversidad en Superficies, según este índice fue de 1,5 .

Se encontró diferencia estadísticamente significativa $(\mathrm{p}<0.05)$ analizando la sumatoria de aislamientos de UTI de ambas estaciones, respecto de Bl.
Aplicando el test de Student a los géneros que aparecieron en UTI, el único que arrojó un $\mathrm{p}<0,05$ fue Aspergillus.

No se encontró diferencia significativa entre los aislamientos de otoño y primavera en ninguno de los tres puntos de muestreo (UTI, Sup. y Bl).

\section{DISCUSION}

Hoy el control de calidad del aire de ambientes cerrados se considera de sustancial importancia porque muchas personas pasan la mayor parte de su tiempo en ellos. En los espacios confinados, los bioaerosoles constituyen un gran problema para aquellos sujetos que sufren enfermedades respiratorias o alérgicas. La calidad del aire de los ambientes confinados es un problema de salud pública con carácter social y económico porque afecta la integridad del individuo, la calidad de vida y la eficiencia de los trabajadores.

Si bien la interpretación de los resultados en los muestreos de aire es frecuentemente problemática por una multiplicidad de factores, los resultados obtenidos en el presente estudio demuestran la existencia de mohos y levaduras en mayor cantidad que la esperada en el tipo de sala muestreada. Los valores establecidos por los departamentos de salud de países americanos y europeos indican que la cantidad de $\mathrm{UFC} / \mathrm{m}^{3}$ no debe sobrepasar las $70 \mathrm{UFC} / \mathrm{m}^{3}$ en salas de alto riesgo, como quirófanos en general, ni las 400 en las salas de internación común (28). 
El ISPEL de Italia (Istituto Superiore per la Prevenzione e la Sicurezza del Lavoro), establece niveles aún menores (9). Los datos presentados son el promedio de lo hallado y teniendo en cuenta los inconvenientes detallados en resultados, es fácil deducir que la contaminación era muy alta.

La frecuencia de aislamientos y la diversidad en UTI fue considerablemente mayor que en Bl, en ambas estaciones. Este es un resultado inverso al esperado y probablemente se deba a la humedad generada por las pilas de lavado, los nebulizadores, los respiradores de asistencia mecánica y posiblemente asociado al funcionamiento del sistema acondicionador de aire dentro de la sala.

Para obtener una información fidedigna se recomienda la utilización de métodos de muestreo combinados, ya que no es posible recoger y evaluar todos los componentes de la biota fúngica utilizando un único método de muestreo $(1,12,13)$. En este caso la aplicación del método del hisopo y el SAS, permitieron la detección de una diversidad mayor.

Se eligió realizar el trabajo en las estaciones de otoño y primavera, porque en esta región son los períodos más y menos lluviosos respectivamente. En otoño se obtuvo mayor carga fúngica que en primavera, hecho que concuerda con lo registrado para el aire exterior de las ciudades de Corrientes, de Resistencia y de Córdoba (Argentina) $(11,29)$. Recordando que las grandes diferencias metodológicas y geográficas dificultan la comparación de resultados.

El horario de muestreo se determinó buscando el momento con menos movimiento de personal dentro de la sala y evitando los horarios de visita. Además, según lo expuesto en otros trabajos, a mediodía la concentración fúngica es mayor (13).

Para establecer el volumen de aire que permitiera el recuento de colonias se efectuaron colectas de prueba con 400, 300 y 200 litros. Se eligió muestrear con 200 litros, dado que en todos los muestreos con 400 y 300 litros resultó imposible realizar el recuento e identificación de las colonias, porque en el punto de impacto del flujo de aire crecían hasta tres colonias una sobre otras.

Los géneros más frecuentes de este monitoreo, Penicillium, Aspergillus y Cladosporium, se encuentran dentro de los microorganismos normalmente presentes en el aire y las superficies (10). Resultados similares fueron obtenidos por De Barros et al., 1990 y Centeno \& Machado, 2004 en Brasil y Venezuela respectivamente $(30,31)$. Su sola presencia no representa riesgo para el hombre, si bien, cargas elevadas de estos agentes en ambientes internos pueden generar situaciones riesgosas (10).

Alternaria alternata complex, A. niger, Chr. sitophila (similis), C.cladosporioides, Curv. lunata y las levaduras se presentaron con constancia en otoño y primavera en los tres puntos de muestreo. Estas especies son hongos cosmopolitas que se adaptan a diversas situaciones ambientales, pero con mayor presencia en zonas tropicales y subtropicales como el área estudiada.

El desarrollo de Chrysonilia sitophila (similis), el «moho rojo del pan», nos impidió el recuento de colonias en una oportunidad. Este hongo es conocido por su crecimiento explosivo e invasor, que en pocos días puede contaminar los cultivos de un laboratorio. Además de la contaminación de cultivos y alimentos, en el ambiente hospitalario, su presencia es de riesgo por estar relacionado con afecciones en pacientes con perforaciones corneales o alteración de las barreras por cirugías, también se lo ha encontrado en catéteres venosos o peritoneales $(18,19)$.

El género Aspergillus es ubicuo, abunda en suelo, aire, granos almacenados y materia orgánica en descomposición $(18,32)$. El hombre puede inhalar abundantes conidios de Aspergillus diariamente, y de hecho lo hace, pero el individuo inmunocompetente raramente enferma, ya que estos propágulos son eficientemente eliminados por el sistema inmunitario celular $(33,34)$. Por el contrario, los pacientes con alguna inmunodepresión o en estado crítico se infectan con frecuencia con estos hongos. El género Aspergillus comprende más de 200 especies de las cuales unas 20 han sido reconocidas como agentes oportunistas. Entre ellas A. fumigatus complex, A. flavus, A. niger, A. terreus y $A$. nidulans, son causantes de una amplia gama de trastornos, siendo la aspergilosis invasora la más temida por su alta morbilidad y mortalidad $(35,36$, 37).

La presencia de $\boldsymbol{A}$. fumigatus en ambientes cerrados, más aún en salas con pacientes de alto riesgo, se considera inaceptable (38). Esta especie es el mayor causante de colonización fúngica e infección invasora $(39,40)$. La infección por $\boldsymbol{A}$. fumigatus no es la más frecuente en el ambiente hospitalario pero suele ser una de las más graves.

La aparición de $\boldsymbol{A}$. flavus en UTI, es un grave factor de riesgo. Esta especie es la segunda causa de aspergilosis invasora y uno de los agentes más frecuentes de infección superficial. Brotes de aspergilosis que implican piel, mucosa oral o tejidos subcutáneos se asocian más a esta especie que al resto de los Aspergillus (36,37).

A. ochraceus es relativamente raro dentro de edificios, pero su presencia en los ambientes internos se considera un problema muy serio porque en el polvo de casas colonizadas con esta especie se detectó presencia de ocratoxinas (41).

A. sydowii es un hongo mesofílico, saprófito del suelo, queratinolítico, de distribución mundial con gran capacidad adaptativa, ya que puede infectar tanto a corales 
abanico (Acabaria splendens) en el mar, como al ser humano. En el hombre ha sido informado como agente de aspergilosis invasora, queratomicosis y onicomicosis (18). En este caso sólo apareció dentro de la sala, tal vez relacionado con su capacidad de colonizador primario de materiales como: fibra de algodón, madera, plásticos, etc. (41).

A. parasiticus es un moho del suelo conocido por producir aflatoxinas, considerado solo como alergénico hasta que Salim et al. (2008), informaron sobre un caso de aspergilosis sinusal en un niño inmunocomprometido en el norte argentino (34), lo que hace más destacable aún su hallazgo en la superficie del espacio estudiado.

Penicillium fue el género hialino aislado con mayor frecuencia y con mayor diversidad de especies. $\mathrm{Su}$ presencia es destacable por la capacidad toxicogénica de algunas de ellas y porque ha sido informado especialmente como patógeno oportunista de heridas quirúrgicas y abrasiones importantes de la piel, afecciones frecuentes de pacientes internados en la sala monitoreada $(18,42)$. $\boldsymbol{P}$. brevicompactum apareció exclusivamente en primavera, tanto en UTI como en las superficies, puede causar una enfermedad multisistémica, a veces difícil de diagnosticar, relacionada especialmente a sus metabolitos tóxicos $(10$, 41).

Curvularia es el género de hongos dematiáceos hallado con mayor frecuencia después de Cladosporium. La importancia de su presencia radica en los informes sobre endocarditis posteriores a cirugías cardíacas, enfermedades diseminadas por lesiones cutáneas, curvulariosis pulmonar y metástasis cerebral, diagnosticadas en pacientes internados en salas de riesgo $(18,42,43,44)$.

Desde la perspectiva de salud pública, la presencia Stachybotrys atra es un alerta, porque es un indicador de la presencia de focos de humedad y porque las consecuencias de la inhalación de sus propágulos, que portan metabolitos tóxicos (Satratoxina G y H), son graves. Se han informado casos de enfermedad pulmonar grave asociadas a Stachybotrys en niños $(6,41)$.

Los siguiente puntos destacan la importancia del control del aire en la sala estudiada: 1) el número UFC $/ \mathrm{m}^{3}$ encontrado excedió considerablemente los parámetros establecidos; 2) A. fumigatus y Stachybotrys atra, se hallaron en baja cantidad y son especies consideradas inaceptables en ambientes cerrados, más aun en áreas críticas; 3) Algunas de las especies encontradas son agentes de micosis oportunistas o tienen la capacidad de elaborar metabolitos tóxicos, por lo que su sola presencia indica un factor de riesgo para los pacientes internados.

El hecho de que se haya detectado mayor frecuencia de aislamientos y diversidad de especies en la sala de UTI que en el Bl puede atribuirse a que: 1) los filtros de aire no eran los adecuados y además estaban deteriorados, 2) el personal y las visitas ingresaban sin la vestimenta adecuada, 3) la puerta de acceso a la sala estaba generalmente abierta, 4) en la sala hay cinco piletas de lavado. Recientemente la atención se ha dirigido hacia el rol que tiene el agua de los hospitales como posible fuente de hongos $(45,46,47)$. Se han recuperado hongos oportunistas de piletas y duchas en varios hospitales de USA. En Canadá un estudio reveló que el $94 \%$ de las muestras de agua tomadas dentro del hospital contenían hongos filamentosos. A. fumigatus fue recuperado del $49 \%$ de las muestras de agua tomadas del grifo de la unidad de trasplante de médula pediátrica. A. terreus, $A$. niger y especies de Fusarium se han aislado de duchas (45). El descubrimiento del agua de los hospitales como fuente de hongos filamentosos sugiere una nueva ruta de transmisión de las micosis invasoras. Bien puede ser que los conidios presentes en el agua sean aerosolizadas durante actividades, como ser la higienización de los pacientes.

La información proporcionada por este estudio determinó que en UTI se intensificaron la limpieza del sistema de aireación y las medidas para diminuir la carga fúngica ambiental.

Teniendo en cuenta que el área hospitalaria estudiada es un ambiente que debe permanecer libre de cualquier contaminación microbiana y, que los resultados obtenidos demuestran la presencia de mohos y levaduras que pueden ocasionar infecciones graves, se sugiere la planificación e implementación de medidas de control higiénico tales como, la desinfección adecuada de las áreas críticas, la instalación de sistemas acondicionadores de aire con filtros apropiados y mantenimiento frecuente, y la evaluación periódica del grado de contaminación de los ambientes, con el fin de reducir las fuentes contaminantes $\mathrm{y}$ así prevenir infecciones fúngicas oportunistas.

\section{REFERENCIAS}

1. Zumbado, H. (2004). Epidemiología y Control Microbiológico de Infecciones. En Manual de Procedimientos en Microbiología Clínica, Editor: Henry D. Isenberg.

2. Martí Solé, C.M.; Alonso Espadalé, R.M.; Constans Aubert, A. (2005). NTP 335: Calidad de aire interior: evaluación de la presencia de polen y esporas fúngicas. En Manual de Microbiología $4^{\mathrm{a}}$ edición. http://www.mtas.es/insht/ntp/ntp_335.htm

3. Hirst, J.M. (1995). Bioaerosols: Introduction, retrospect and prospect. En: Bioaerosols Handbook. Ed. Cox, C.S. and Wathes C.M. Lewis Publishers, Boca Raton, USA. pp. 1-10

4. Crook, B. \& Sherwood-Higham, J.L. (1997). Sampling and Assay of Bioaerosols in the Work Environment. J. Aerosol. Sci. 28:417-426

5. Flannigan, B. \& Miller, D. (1994). Health implications of fungi in indoor environments - an overview. En: Samson RA; Flannigan B; Flannigan ME; Verhoeff AP, Adan OCG, Hoekstra 
ES, eds. Health implications of fungi in indoor environments. Air quality monographs 2:3-11

6. Miller, D.J.; Rand, T.G \& Jarvis, B.B. (2003). Stachybotrys chartarum: cause of human disease or media darling? Med. Mycol. $41: 271-291$

7. Spieksma, F.T.; Kramps, J.A.; van der Linden, A.C.; Nikkels, B.H.; Plomp, A.; Koerten, H.K.; Dijkman, J.H. (1990). Evidence of grass-pollen. Clin. Exp. Allergy 20:273-80

8. Streifel, A. (1996). Hospital Epidemilogy and Infection Control. En: Design and Maintenance of Hospital Ventilation Systems and the Prevention of Airborne Nosocomial Infectios. Glen Mayhall Ed. USA.

9. Alfano, V.; Visciano, A.; Liguori, G. (2007). Aspetti pratici del monitoraggio microbiologico ambientale. Biologia Clinica $2: 30-34$

10. UNICHIM. (2009) Manuale N. 203. Rischio biologico in ambienti «indoor». Inquadramento della problematica e strategia di controllo e prevenzione. Associazione per l'Unificazione nel Settore dell'indutria Chimica. Federata all'UNI. Milano.

11. Esquivel, P.; Mangiaterra, M.; Giusiano, G.; Sosa, M.A. (2003). Microhongos anemófilos en dos ciudades del nordeste argentino. Boletín Micológico 18:21-28

12. Martí Solé, M.C. (1993). NTP 299: Método para el recuento de bacterias y hongos en aire.http://www.mtas.es/insht/ntp/ ntp_299.htm.

13. Rojas, T.I.; Martínez, E.; Aira, M.J.; Almaguer, M. (2008). Aeromicota de ambientes internos: Comparación de métodos de muestreo. Boletín Micológico 23:67-73

14. Nelson, P.; Tousson, T.; Marasas, F. (1983). Fusarium species. An illustrated Manual for identification. The Pennsylvania State University Press. University Park. London.

15. Gams, W. (1971). Cephalosporium-artige Schimmelpilze Hyphomycetes. G Fisher Verlag. Stuttgart.

16. Onions, A.; Allsopp, D. \& Eggins, H. (1981). Smiths's introduction to industrial Micology, $7^{\mathrm{a}}$ ed. John Wiley \& Sons. New York.

17. Von-Arx, J.A. (1981). The genera of fungi sporulating in pure culture. $3^{\mathrm{a}}$ ed. Edit J. Crame. Vaduz.

18. De Hoog, G.; Guarro, Gené, J. \& Figueras, M.J.(2000). Atlas of Clinical Fungi, $2^{\mathrm{a}}$ ed. The Netherlands: Centraalbureau voor Schimmelcultures, Utrecht, The Nederlands, Universitat Rovira i Virgili Reus, España.

19. Samson, R.; Hoekstra, E.; Frisvad, J.C.\& Filtenborg, O. (2000). Introduction to food and airborne fungi. Utrcht. Centraalbureau voor Shimmalcultures. The Netherlands.

20. Rifai, M.A. (1969). A Revision of the Genus Trichoderma. Mycological Papers No 116

21. Bissett, J. (1991). A Revision of the Genus Trichoderma. H. Infrageneric classification, Can. J. Bot. 69:2357-2372

22. Piontelli, E. \& Toro, M.A. (2003). Manual de identificación para microhongos communes en alimentos. Universidad de
Valparaíso. Escuela de medicina. Cátedra de Micología. Valparaíso.

23. Sivanesan, A. (1987). Graminicolous species of Bipolaris, Curvularia, Drechslera, Exserohilum and their teleomorphs. Mycological papers, $\mathrm{N}^{\circ}$ 158. C.A.B. Internatinal Mycological Institute.

24. Pitt, J.I. (1986). A laboratory guide to common Penicillium species, Reprinted. Australia: Commonwealth Scientific and Industrial Research Organisation, Division of food Reserch.

25. Ellis, M.B. \& Ellis, J.P. (1987). Microfungi on land plants. An identification handbook. Croom Helm. Australia.

26. Klich, M.A. \& Pitt, J.I. (1988). A laboratory guide to common Aspergillus species and their teleomorphs. Reprinted. Commonwealth Scientific and Industrial Research Organization, Division of food Processing. Australia.

27. Carmichael, J.; Kendrick, B.; Conners, I. \& Sigler, L. (1980). General of Hyphomycetes. The University of Alberta Press. Canada.

28. Cerminara, R.H. (2000). Control del aire en los Hospitales. www.enfermeria21.com/listametas.doc

29. Quiroga de Pascual, R.L. \& Nobile, R. (1985) Incidencia de hongos ambientales durante un año en la ciudad de Córdoba. Rev. Argent. Micol. 8:16-22

30. De Barros, M.G.; Cavalcanti, M.A.; Massa Lima, D.M; Fernández, M.J.S. (1990) Fungos isolados do ar e do piso de ambientes fechados do Hospital Escola da Universidad Federal de Pernambuco, Recife, Brasil-I. Boletín Micológico 5:57-67

31. Centeno, S. \& Machado, S. (2004). Evaluación de la micoflora aérea en las áreas críticas del Hospital Principal de Cumaná, estado Sucre, Venezuela. Departamento de Bioanálisis, Universidad de Oriente, Núcleo de Sucre, Cumaná, Venezuela. Investigación Clínica. Vol. 45 № 2. Maracaibo.

32. Piontelli, E. (2008). Aportes morfotaxonómicos en el género Aspergillus Link: Claves para las especies ambientales y clínicas más comunes. Boletín Micológico 23:49-67

33. Pérez, F.; Opazo, H.; Cruz, R. \& Piontelli, E. (2006) Reporte clínico. Diagnóstico endoscópico e histológico de aspergillosis sinusal no invasiva en paciente inmunocompetente. Boletín Micológico 21:85-89

34. Salim, R. \& Runco, R. (2008) Aspergilosis sinusal no invasiva por Aspergillus parasiticus en niño inmunocomprometido. Boletín Micológico 23:1-7

35. Samson, R.A; Flannigan, B.; Flannigan, M.E.; Verhoeff, A.P.; Adan, O.C.G; Hoekstra, E.S. (1994). Health implications of fungi in indoor environments. Air quality monographs. Vol.2. Elseiver. Amsterdam.

36. Cruz, R.; Barthel, M.; Piontelli, E. \& Fernández, G. (2005) Reportes clínicos. Infección Rinosinusal probada por Aspergillus flavus y probable infección pulmonar por Emericella nidulans en pacientes inmunodeprimidos. Boletín Micológico 20:109-115

37. Hedayati, M.T.; Pasqualotto, A.C.; Warn, P.A.; Bowyer, P.; Denning, D.W. (2007) Aspergillus flavus: human pathogen, 
allergen and mycotoxin producer. Microbiology 153: 1677-1692

38. Department of National Health and Welfare (DNHW) (1987) Exposure Guidelines for Residential Indoor Air Quality, DNHW, Ottawa, Canada.

39. Rippon, J.W. (1988) Aspergilosis. En: Tratado de Micología Médica. WB Saunders, 3ra ed., Philadelphia 1988, pp. 668-703

40. Kwon-Chung, K.J. \& Bennett, J.E. (1992) Aspergilosis. En: Kwon-Chung KJ, Bennett JE. Medical Micology. Lea \& Febiger. Pensilvania. pp. 201-247

41. Fog Nielsen, K. Mould growth building materials Secondary metabolites, micotoxins amd biomarkers. Biocentrum-DTU Technical University of Denmark. Lyngby 2002)

42. Richardson, M.D. \& Warnock, D.W. (1998) Fungal Infection. Diagnosis and Management. Blackwell Science. 2nd edition. Berlin.

43. Smolinski, K.N.; Shah, S.S.; Honig, P.J.; Yan, A.C. (2005). Infecciones cutáneas fúngicas neonatales. Curr. Opinion Pediat. 17:486-493
44. Casasco, J.P.; Martinez, D.; De Bonis, C.; Napolitano, N.; Berner, S. (2008). Curvularia lunata: presentación atípica con compromiso del sistema nervioso central. Reporte de un caso. Rev Argent. Neurocir. vol.22, n.3, pp. 0-0. http:// www.scielo.org.ar/pdf/ranc/v22n3/v22n3a15.pdf

45. Warris A.; Voss A. \& Verweij, P.E. (2001) Hospital sources of Aspergillus species: New routes of transmission? Rev. Iberoam. Micol. 18:156-162

46. Nagy, L.A. \& Olson, B.H. (1982) The occurrence of filamentous fungi in drinking water distribution systems. Can. J. Microbiol. 28:667-671

47. Paterson, R.R.M.; Kelley, J. \& Gallagher, M. (1997) Natural occurrence of aflatoxins and Aspergillus flavus (Link) in water. Lett. Applied Micobiol. 25:435-436 